\title{
PSYCHOPHYSIOLOGICAL METHODS AND CRITERIA FOR THE SELECTION OF INDIVIDUAL METERED LOADS IN ATHLETES OF TAEKWONDO SECTION
}

\author{
Ohromiy G. V. ${ }^{1}$, Makarova N.U. ${ }^{2}$, Kasyuha A.M. ${ }^{1}$ \\ Ukrainian State University of Chemical Technology

$$
\mathrm{MEDA}^{2}
$$

Annotation. Purpose: scientific rationale approaches Express-diagnostics for athletes of taekwondo section by psychophysiological parameters. Material: Total surveyed 84 people, including 68 boys and 16 girls. Were defined backup capabilities, exercise tolerance and selection of adequate individual dose of loads in preparation for the international competition, participants - taekwondo athletes. Was attended by representatives of Ukraine, Russia and Lithuania. Age groups of participants: 6 - 16 years. Athletes have different levels and different schools of training. Results: evaluation of exercise tolerance and definition of an adequate level of motor mode in taekwondo athletes in terms of the difference of lability (DL) before and after training, revealed: high and very high nervous system (NS) lability. These figures correspond to high and very high tolerance to physical loads. The respondents who had difference of liability (DL) $<0$, were well-trained athletes and their high points of strength of nervous system testified about good backup capabilities (withstand great and continuous loads). In $78 \%$ of the tested load was chosen adequately. Less than $20 \%$ of the respondents noted the average backup capabilities. They need individual correction dose loads to increase adaptive capacity. Insignificant $10 \%$, the share of respondents had low exercise tolerance at the average values NS strength, which indicates a good backup capabilities. The direct dependence: respondents with high levels on state of health, activity and mood - low levels of anxiety, frustration, aggression and rigidity. Conclusions The proposed optimized approach Express-diagnostics according to psychophysiological parameters allow you to determine the level of physical endurance, speed adaptation athletes in the sections taekwondo and adequate response to the loads.

Keywords: taekwondo, exercise, tolerance, express, diagnostics, physiological, parameters.

\section{Introduction}

According to official statistics, physical inactivity among young people is $60-80 \%$ of the required level [http://zakon2.rada.gov.ua/laws/show/963\%D0\%B0/98]. It is proved that physical endurance depends not only on the physical development of the person, but also on psycho-physiological properties of the nervous system [10-12]. But, despite the rather large contribution of scientists to the problem of improving the health of the younger generation, and improve performance in athletes, not developed standardized criteria for determining individual optimal physical activities, taking into account these parameters [7-9].

Wide promotion of healthy lifestyles, aims population of all ages to the sport. However, over the past $1.5-2$ decades has increased significantly the number of cases of serious deviations in the health of athletes $[1,6]$.

According to Erich Doyzer modern great sport has little to do with the health of an athlete and is now becoming more and more justified. Sports-medical practice confirms this position [13-15]. In today's sports coaches considered only the physical health athlete, and not always taken into account the psychophysiological status at the time of expansion of individual loads (Report on the situation in the field of non-communicable diseases in the world, 2010 Executive Summary. - WHO: Geneva, 2011. - 20 p. ) [1-5]. To achieve performance in sport is not always taken in consideration individual psychological characteristics and physiological capabilities athlete that has defined the goal of our study.

Purpose, tasks of the work, material and methods

Objective: scientific rationale approaches "Rapid diagnostic individually-dosed physical load" for Taekwondo athletes section on physiological parameters.

Objectives and methods. The main methods used are:

1. To develop criteria for "quick control" of individual optimal dose loads during training, and in the period of intensive training regime, on the eve of the competition, Taekwondo, used the technique of diagnosing the properties of the nervous system on psychomotor performance E. P. Ilin modified by G. V. Ohromiy.

Determined by the lability of the nervous system (LNS) - the rate of reaction to the load and the strength of the nervous system (SNS) - spare capacities athlete, that is, the ability to hold the pace and workload [Pat. 75615 A61B 5/16 (2006.01)/ u 2012 05608; Pat. 77886 A61B 5/16 (2006.01)].

The essence of the method of assessment of lability is to perform fast stereotyped movements with the hand with drawing dots on paper with a pencil (tapping). Tapping can be measured at an optimal pace of rapping, i.e. when the rhythm, preferably for the test, and at maximum pace. Frequency is a measure of the maximum tapping energetic component activity, manifested in the physical endurance fatigue. The present study used the maximum dimension tapping as described below.

In the study of athletes during the passage of stress tests tapping was measured twice - before and after a recovery period of stress test. To conduct tapping test used special forms depicting 6 squares of $5 \times 5 \mathrm{~cm}$ and arrows indicating the direction of the transition. Purpose of assignment formulated as applying a maximum possible number of dots within 30 seconds, each square must be completed within 5 seconds. Before starting the tests you need to put a 
pencil in front of the first square and the command "Go!" To move it to the first square and begin a quest. Transition to the next square is made every 5 seconds at the command "Go!" Conducting the survey.

Processing of test results included counting the number of pixels in each box, and plotting efficiency: the abscissa 5-second intervals, and the ordinate - number of points in each square. The resulting variants dynamics maximum rate may be divided into the following types (Fig. 1):

\section{METHOD}
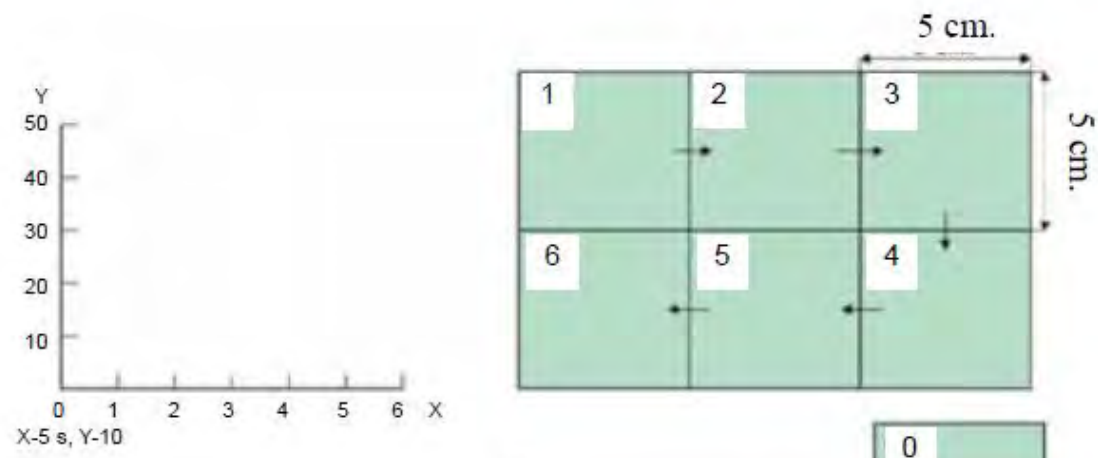

Y
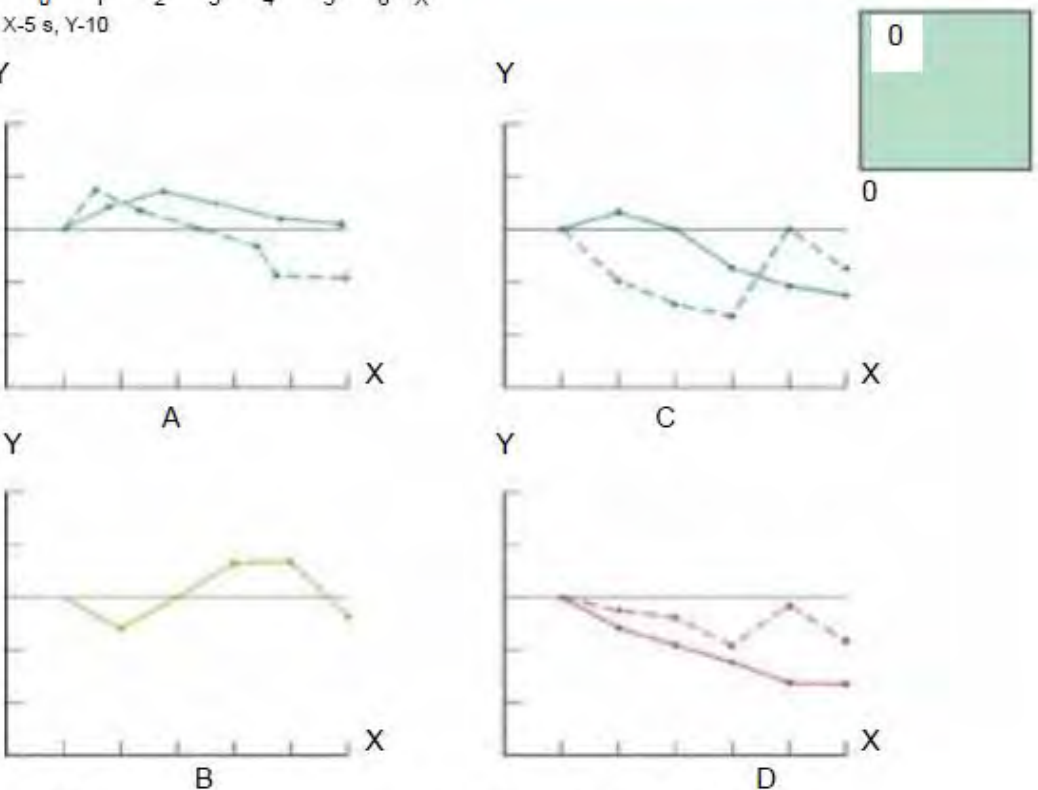

Y

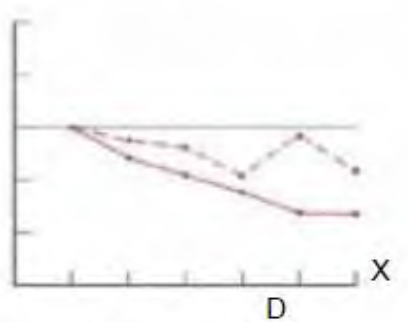

C - medium, weak

A - strong

D - weak

Fig. 1 Types of dynamic maximum rate movements

Conclusion on the lability of sensorimotor analyzer case on the total amount of points. An estimation of the lability of the nervous system, the number of points is translated into points by the following table (Table 1).

Table 1

Definition of lability by symbols and points

\begin{tabular}{|c|c|c|c|c|c|c|c|c|c|c|}
\hline Number of dots & $<74$ & $75 \square 129$ & $130 \square 151$ & $152 \square 162$ & $163 \square 172$ & $173 \square 183$ & $184 \square 195$ & $196 \square 204$ & $205 \square 210$ & $>210$ \\
\hline Points & 1 & 2 & 3 & 4 & 5 & 6 & 7 & 8 & 9 & 10 \\
\hline Lability & & Low & & & Medium & & & High & & Very High \\
\hline
\end{tabular}

Proposed author's index (to align the system of points from 1 to 10 ), calculated by the formula, in preliminary studies was more informative than the simple sum of the deviations as E. P. Ilin described:

$$
\text { strength indicator }=5+\frac{S_{2}+S_{3}+S_{4}+S_{5}+S_{6}+5 \cdot S_{1}}{S_{1-6}} \cdot 10
$$

$\mathrm{S}_{1}, \mathrm{~S}_{2}, \mathrm{~S}_{3}, \mathrm{~S}_{4}, \mathrm{~S}_{5}, \mathrm{~S}_{6}$ - the number of points in squares 1-6 respectively, $\mathrm{S}_{1-6}$ - the total number of points. 
In the process of tapping multiple testing in studies from the author the idea to automate the process of evaluation of the functional state of the central nervous system with a special device. The idea of the author has been implemented development team of the company «Comet» in Dnepropetrovsk. As a result, the device is designed to automate the process of Tapping Test and program for processing the test results issued patent. This device was tested and applied in the process of further research of the author.

1. By the method of Eysenck H. were studied self esteem mental states: aggressiveness (characteristic of the subject, reflecting its predisposition to behavior which is intended to anger); Frustration (a condition resulting from failures); rigidity (unwillingness to change the program of action in accordance with the new situational demands); anxiety (individual psychological peculiarities manifested in human propensity often experience intense anxiety over relatively small occasions).

2. Diagnosis of health, activity and mood (SAN) athlete during training and before the competition.

Materials research. The study was conducted on the basis of the Camp «Eaglet», located on the Black Sea coast of Feodosia (Crimea). Participants athletes of different age groups from 6 years - 16 years. Boys and girls have different levels of training. Much importantly, the presence of coaches from different schools and approaches to the training process. Were presented to countries such as Ukraine, Russia, Lithuania. Exercise duration 1.5 hours. The study was conducted over 4 days using different methodologies. Total surveyed 84 people, including 68 boys and 16 girls.

\section{Results of the researches}

Evaluation of exercise tolerance (ET) was determined by the difference of lability (DL) of the nervous system (NS) before and after exercise and the deviation from the average figure ( 5 points). for the index:

$\mathrm{DL}>1$ - tolerance is reduced $(36-50 \%)$;

$\mathrm{DL}=1$ - tolerance moderately reduced $(51-70 \%) ;$

$\mathrm{DL}=0$ - tolerance maintained $(71-85 \%)$;

$\mathrm{DL}<0$ - tolerance is high $(86-100 \%)$.

The results of evaluation exercise tolerance and to determine the adequacy of the amount of motor mode on the results of radar identified:

1. After physical loads (PL) (workout and exercise) in $24 \%$ of cases, there is a high ( $8 \pm 1$ point) and in $7 \%$ of cases is very high $(9 \pm 1$ point) the lability of the NS. These figures correspond to high ( $86 \%$ or more) and very high (up to $100 \%$ ) tolerance PL. The calculated rate DL $<0$ for these respondents fluctuated in the range of (1) to (4) points, which indicates a good fitness of this share of athletes.

Detected in this category of persons and a high ball strength NS. It ranged from $8 \pm 1$ point to $9 \pm 1$ point. These athletes good backup capabilities. They can withstand large in magnitude and long load.

2. Preservation of tolerance (71-85\%), DL $=0$, was detected in $40 \%$ of cases and is $4 / 10$ of the total number of patients. Average SNS corresponded $6 \pm 1$ score. For this category of individual athletes adequately matched load.

3. Athletes (19\%) with moderately reduced tolerance $(51-70 \%)$, the difference lability $(\mathrm{DL})=1$. Identification of SNS indicators in some cases had minor deviations as downward ( $4 \pm 1$ point) and upward ( $5 \pm 1$ point) from the standard average value equal to the SNA 5 points. This category of middle backup capabilities. They are at risk, in need of correction of individual dosage burdens and increase adaptive capabilities.

4. 1/10 share of respondents had low exercise tolerance (36-50\%), DL $>1$. However, in determining the SNS in more than half of the respondents identified SNS indicators corresponded to the average of ( $5 \pm 1$ point), and in some cases above the average value $(6 \pm 1$ point). This suggests that athletes have good backup capabilities, but the approach should be individualized. Each change of load, in our opinion, should be adapted and secured in a longer time interval.

Developed model «Method of determining human tolerance to stress» on the difference of lability (DL) is unified and can be applied to the definition of tolerance and physical stress (Table 2).

To determine the tolerance to physical loads on the main horizontal scale models determine baseline lability of the nervous system in the test scores using «Tapping-test». After a given physical activity carried out follow-up study of the nervous system lability test (using «Tapping-test») and by determining the lability of the nervous system in points, find value in the vertical column corresponding to the first indicator. Determine the difference between the baseline lability index and after exercise by substituting the parameters in the horizontal and vertical column, respectively. Based on these factors determine the tolerance to physical exertion.

Example. Carry out «Tapping-test». Indicator lability of the nervous system to the load - 156 points. By main scale model «Method of determining human tolerance to stress» is defined baseline lability of the human nervous system, equal to -3 points (156 dots). After exercise is carried out repeatedly «Tapping-test». Indicator lability of the nervous system after exercise - 178 dots. The vertical scale in the first indicator (156 dots, 3 points) define indicator lability human nervous system after exercise equal - 5 points (178 dots). Correlate human lability of nervous system before and after physical load on a scale determined by the index of tolerance tolerance, it is equal to DL $<0$. Conclusion: exercise tolerance is high $86-100 \%$.

Model helps determine the speed of reaction to mental and physical stress in the educational process, the switching speed of the attention of students on various activities in learning environments in higher education. 
Model «Method of determining human tolerance to stress»

Table 2

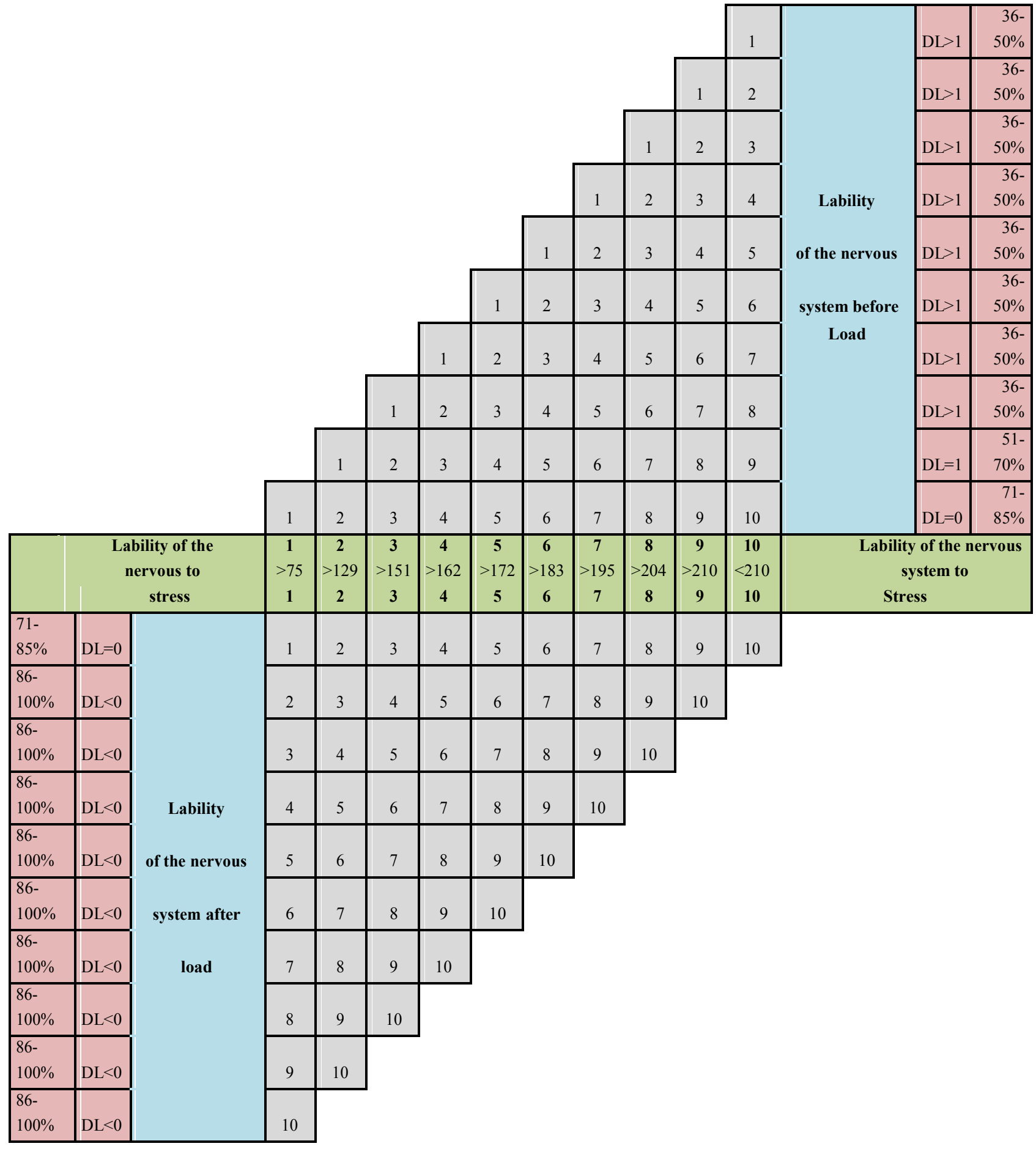

\begin{tabular}{|c|c|c|}
\hline DL $>1$ & Low tolerance to stress & $\mathbf{3 6 -}$ \\
\hline & & $\mathbf{5 0 \%}$ \\
\hline $\mathbf{D L}=\mathbf{1}$ & Moderately low tolerance to stress & $\mathbf{5 1 -}$ \\
\hline & & $\mathbf{7 0 \%}$ \\
\hline DL $=\mathbf{0}$ & Saved tolerance to stress & $\mathbf{7 1 -}$ \\
\hline & & $\mathbf{8 5 \%}$ \\
\hline $\mathbf{D L}<0$ & High tolerance to stress & $\mathbf{8 6 -}$ \\
\hline
\end{tabular}

*DL - Difference of lability 
Determine the tolerance of students to physical stress by index of LNS and SNS can and our model (Table 3) «Method for determining the physical human endurance». In the model of two scales : the left and right scale lability of the nervous system, inside - the scale of nervous system strength and performance of physical activity due in watts (W).

To determine exercise tolerance is diagnosed properties of the nervous system of humans on psychomotor performance by «Tapping-test».

Modeled find lability score score nervous system and nervous system strength. At the intersection of performance and power LNS and SNS define threshold physical load in watts. The corresponding figures for the cycle ergometer.

Example. Carry out diagnosis of nervous system by using «Tapping-test». Indicator lability of the nervous system - 7 points (187 dots), the index of nervous system strength - 3 points . Using model "Method for determining the physical human endurance" find correspondence between the scale and the scale of the lability of nervous system strength. Get the result - the threshold load - 298 watts. You can define the deviation from the mean: median of LNS - 5 points, the mean of SNS - 5 points, the load on the intersection - 330 watts. The difference $330-298=32 \mathrm{~W}$, the deficit threshold of physical activity 32 watts.

Table 3

Model «Method of determination the physical endurance»

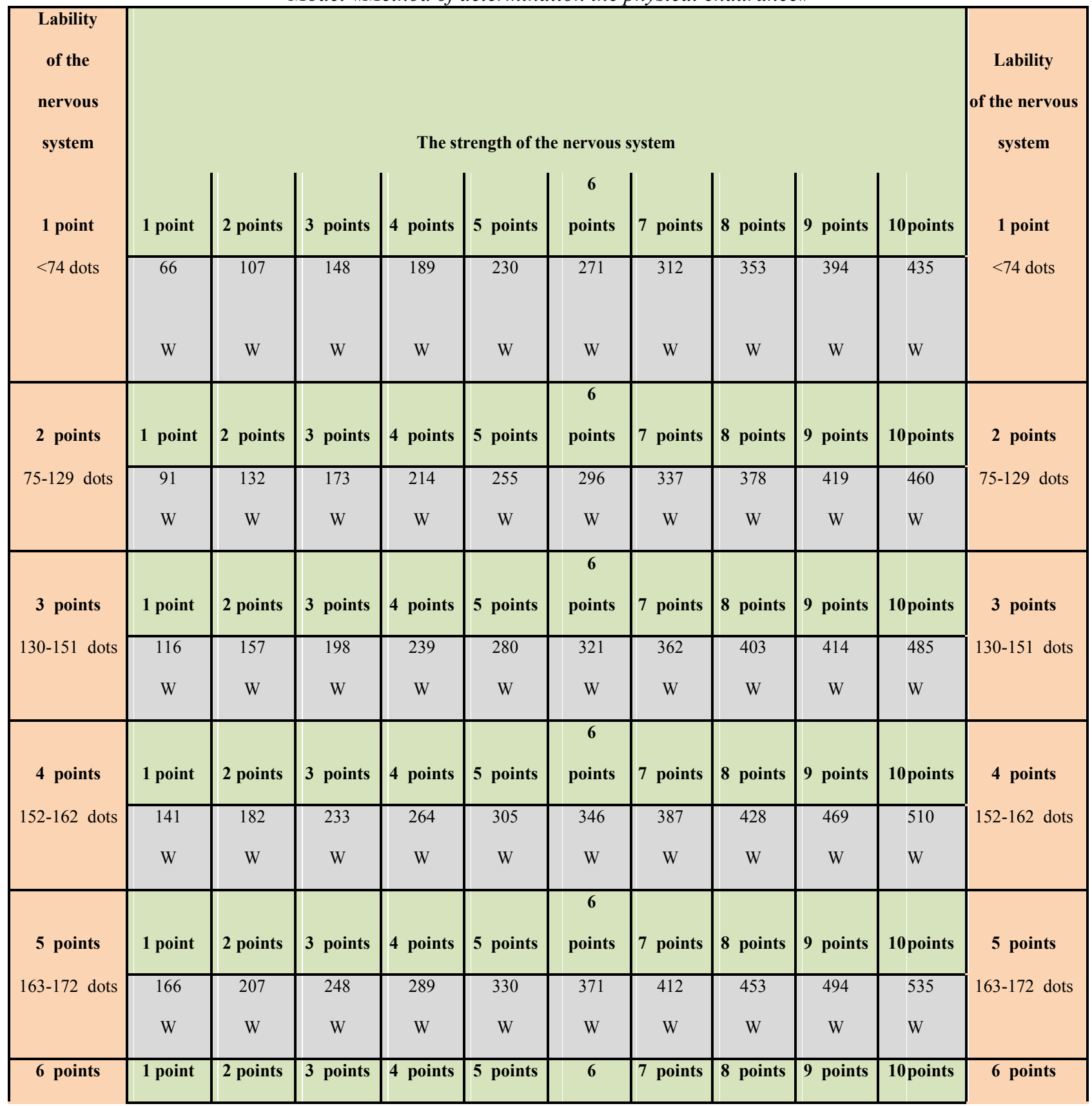




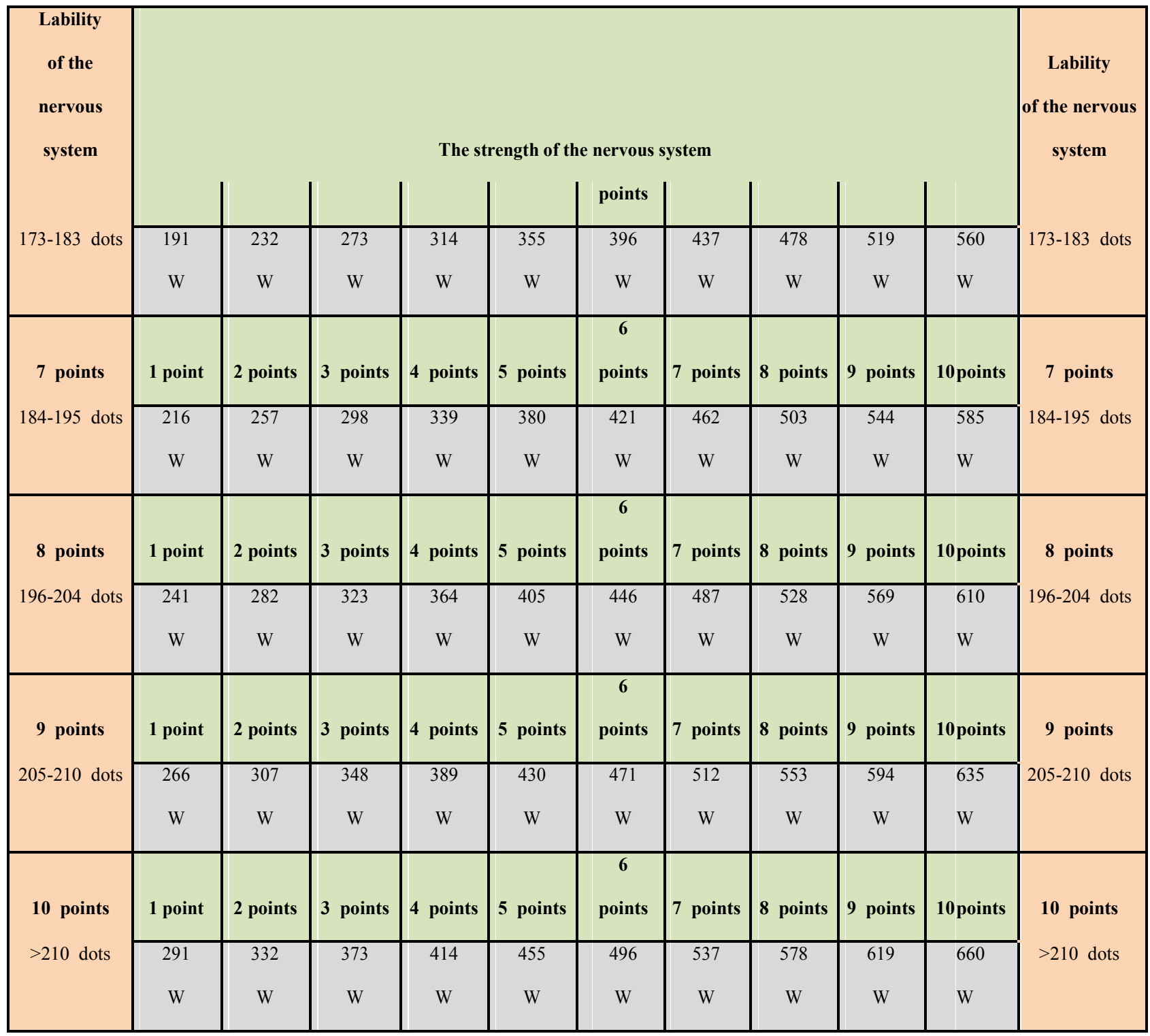

Following the procedure of SAN and the Eysenck tested 33 leading athlete (Table 4). Investigating health, activity and mood to the SAN method we can say that: more than half of the athletes - in $56 \%$ (17 people). Highest rate of health, 31\% (10 people) Average and 13\% (6 people) low. A large proportion of individuals 56\% (17 people). Had high activity, 43\% (14 people). Medium and 1\% (2 people) Low. Extensive evidence of high mood was observed in $69 \%$ (21 people), 30\% (10 people). Medium and 1\% (2 people) Low, which affected the good results in athletes. High and medium level of anxiety among athletes is not revealed. In $100 \%$ of cases diagnosed with low levels of anxiety. Athletes are not noted the poor condition resulting from failure (frustration). Low state of frustration in $99 \%$ (31 people). Cases. In most cases identified aggressiveness lowest 87\% (29 people), Middle 13\% (4 people), respectively. Low rigidity marked almost all the respondents $98 \%$ (30 people).

Table 4

Performance of the SAN and Eysenck methods

\begin{tabular}{|c|c|c|c|c|c|c|c|}
\hline S & A & N & Power & Anxiety & Frustration & Aggression & Rigidity \\
\hline $56 \%$ & $56 \%$ & $69 \%$ & High & $0 \%$ & $0 \%$ & $0 \%$ & $0 \%$ \\
\hline $31 \%$ & $43 \%$ & $30 \%$ & Average & $0 \%$ & $1 \%$ & $13 \%$ & $21 \%$ \\
\hline $13 \%$ & $1 \%$ & $1 \%$ & Low & $100 \%$ & $99 \%$ & $87 \%$ & $98 \%$ \\
\hline
\end{tabular}


Thus, according to the results of the procedure can be controlled run and stabilize the adequacy of training and support activities in the period of training in the sections taekwondo.

\section{Conclusions:}

1. In selecting individual metered loads revealed that the volume of motor mode does not match the criteria threshold load the athlete if the received indicators remain at the same level. Such physical loads, in our opinion, somewhat overstated and will not increase the tolerance to such persons should be a special approach in training.

2. Growth indices lability and strength of the nervous system with repeated measurements testified about correct selection mode of physical loads.

3. Growth performance and reduced lability and strength of nervous system force with repeated measurements suggest proper selection of the mode of exercise. However, the reserve capacity of the organism is very low.

4. Unchanged or decreased lability and strength of nervous system force growth index during the second dimension, in our opinion, evidence of correct selection of individual metered loads and good backup of an athlete.

5. The proposed methodology can be used: to determine the speed of adaptation of athletes in the classroom in the sections, an adequate response to the load; determine the level of physical endurance aggression and rigidity.

Recommendations: on the results of the procedure can be monitored the adequacy and effectiveness of training and supporting activities in the training period and the beginning of the competition in the sections taekwondo. 


\section{References:}

1. Apanasenko G. L. Kniga o zdorov'e [Books on health], Kiev, Medical Book, 2007, 132 p.

2. Balakirieva O. Zdorov'ia ta povedinkovi oriientaciyi ukrayins'koyi molodi [Health and behavior of Ukrainian youth], Kiev, 2005, 256 p.

3. Bezmaternykh L.E., Kulikov V.P. Fiziologiia cheloveka [Human physiology], 1998, vol.3, pp. 79-85.

4. Vojtenko V.A. Zdorov'e zdorovykh [Health of healthy], Kiev, Health, 1991, 187 p.

5. Bresciani G., Cuevas M.J., Garatachea N. Monitoring biological and psychological measures throughout an entire season in male handball players. European Journal of Sport Science 2010, vol.10(6), pp. 377-384. doi:10.1080/17461391003699070.

6. Gloria R., Tatiana D., Constantin R.B., Marinela R., Gabriel M. Study Regarding the Order of Psychological Processes and the Importance of the Psychological Factors of High Performance for the Sports Branch Karate. Procedia - Social and Behavioral Sciences 2013, vol.84, pp. 1228-1232. doi:10.1016/j.sbspro.2013.06.734.

7. Huzar V., Shalar O., Norik A. Relationship as an aspect of psychological climate of women's soccer team. Physical Education of Students 2014, vol.2, pp. 8-12. doi:10.6084/m9.figshare.906307.

8. Kharchenko G. Basic principles of renewal of sportsmen with myofascial by a pain syndrome taking into account the psychological aspect of their rehabilitation. Pedagogics, Psychology, Medical-Biological Problems of Physical Training and Sports 2014, vol.8, pp. 29-33. doi:10.5281/zenodo.9760.

9. Law B., Ste-Marie D.M. Effects of self-modeling on figure skating jump performance and psychological variables. European Journal of Sport Science 2005, vol.5(3), pp. 143-152. doi:10.1080/17461390500159273.

10. Medvidchuk K.V., Korobeynikov G.V., Mazmanian K.R. Formation characteristics of psychophysiological functions in combat sports athletes. International Journal of Psychophysiology 2008, vol.69(3), pp. 247. doi:10.1016/j.ijpsycho.2008.05.144.

11. Omelyanenko V. Express-method of sportsmen's psychological tune-up. Pedagogics, Psychology, MedicalBiological Problems of Physical Training and Sports 2014, vol.6, pp. 37-41. doi:10.6084/m9.figshare.1004938.

12. Oliveira P.A., Araújo D., Abreu A.M. Proneness for exercise, cognitive and psychophysiological consequences of action observation. Psychology of Sport and Exercise 2014, vol.15(1), pp. 39-47. doi:10.1016/j.psychsport.2013.09.008

13. Omelyanenko G.A. The modern state of formed level of readiness of bachelors of physical education and sport to research activity. Pedagogics, Psychology, Medical-Biological Problems of Physical Training and Sports. 2011, vol.1, pp. 98 - 101.

14. Stanger N., Kavussanu M., Willoughby A., Ring C. Psychophysiological responses to sport-specific affective pictures: A study of morality and emotion in athletes. Psychology of Sport and Exercise 2012, vol.13(6), pp. 840848. doi:10.1016/j.psychsport.2012.06.004.

15. Tessier D., Sarrazin P., Ntoumanis N. The effect of an intervention to improve newly qualified teachers' interpersonal style, students motivation and psychological need satisfaction in sport-based physical education. Contemporary Educational Psychology 2010, vol.35(4), pp. 242-253. doi:10.1016/j.cedpsych.2010.05.005. 
Information about the authors:

Ohromiy G.V.: ORCID: http://orcid.org/0000-0002-0631-0443; Ogv3@ mail.ru; Ukrainian State University of Chemical Technology; Gagarin ave. 8, Dnepropetrovsk, 49005, Ukraine.

Makarova N.U.: ORCID: http://orcid.org/0000-0001-5573-9513; makarovanua@mail.ru; MEDA; Gagarin ave. 8, Dnepropetrovsk, 49005, Ukraine.

Kasiuha A.M.: ORCID: http://orcid.org/0000-0002-5006-0955; Sashakasyuga@gmail.com; Ukrainian State University of Chemical Technology; Gagarin ave. 8, Dnepropetrovsk, 49005, Ukraine.

Cite this article as: Ohromiy G. V., Makarova N.U., Kasyuha A.M. Psychophysiological methods and criteria for the selection of individual metered loads in athletes of taekwondo section. Physical education of students, 2014, vol.6, pp. 54-62. doi:10.15561/20755279.2014.0611

The electronic version of this article is the complete one and can be found online at: http://www.sportpedu.org.ua/html/arhive-e.html

This is an Open Access article distributed under the terms of the Creative Commons Attribution License, which permits unrestricted use, distribution, and reproduction in any medium, provided the original work is properly cited (http:// creativecommons.org/licenses/by/3.0/deed.en).

Received: 10.06 .2014

Published: 30.06 .2014 